\title{
Review
}

\section{Engineering of Synthetic Transcriptional Switches in Yeast}

\author{
Masahiro Tominaga ${ }^{1,2}$, Akihiko Kondo ${ }^{1-4}$ and Jun Ishiii, ${ }^{1,2}$ \\ 1 Engineering Biology Research Center, Kobe University, 1-1 Rokkodai, Nada, Kobe 657-8501, Japan \\ 2 Graduate School of Science, Technology and Innovation, Kobe University, 1-1 Rokkodai, Nada, Kobe 657- \\ 8501, Japan \\ 3 Department of Chemical Science and Engineering, Graduate School of Engineering, Kobe University, 1-1 \\ Rokkodai, Nada, Kobe 657-8501, Japan \\ 4 Center for Sustainable Resource Science, RIKEN, 1-7-22 Suehiro, Tsurumi, Yokohama 230-0045, Japan \\ * Correspondence: junjun@port.kobe-u.ac.jp; Tel.: +81-78-803-6356
}

\begin{abstract}
Genetic switches can be utilized for many purposes in synthetic biology including the assembly of complex genetic circuits to achieve sophisticated cellular systems and the construction of biosensors for real-time monitoring of intracellular metabolite concentrations. Although genetic switches have mainly been developed in prokaryotes to date, eukaryotic genetic switches are increasingly being reported as both rational and irrational engineering technologies mature. In this review, we describe genetic switches in yeast based on synthetic transcription factors and/or synthetic promoters. We also discuss directed evolution technologies for the rapid and robust construction of yeast genetic switches.
\end{abstract}

Keywords: genetic switch; yeast; synthetic transcription factor; synthetic promoter; directed evolution

\section{Introduction}

In the field of synthetic biology, it is necessary to develop genetic elements such as promoters and terminators to fulfill the increasing demand for precise control of complex gene expression, which is required for the construction of sophisticated biological systems (1). In particular, genetic switches are required to regulate gene expression in response to intracellular and extracellular stimuli (e.g., metabolites and inducers) and thereby modulate cellular phenotypes via their output (2). To date, genetic switches have been utilized to build inducible expression systems $(3,4,5,6)$, genetic circuits $(7,8,9,10,11)$, and metabolite sensors $(12,13,14,15,16)$. Unfortunately, however, most genetic switches cannot be used directly for synthetic biology applications because of their inappropriate switching properties. For example, such promoters often exhibit detectable activity in an OFFstate (leaky expression), which hampers the expression of toxic proteins (17). Besides, if genetic switches are to be used as elements of a synthetic bioengineering toolbox, they must be designed to respond specifically to the desired target chemical with minimal cross reactivity, no leakiness, and sufficient output when activated; otherwise, the construction of complex, higher-order, genetic circuits, especially with layered logic gates, will fail (18).

Endogenous systems such as the Gal4 transcriptional switch $(3,4,5)$ and G-protein coupled receptor systems (19) can be used as genetic switches in yeast. Alternatively, genetic switches can be artificially created using heterologous, ligand responsive, DNA binding proteins, exemplified by bacterial transcription factors (TFs), which can be used to control promoter activity. Tet-ON and Tet-OFF systems that regulate gene expression in response to a small molecule, doxycycline (Dox), were first described as synthetic genetic switches for mammalian systems (20) and subsequently used in various fungi (21, $22,23)$. Yeast genetic switches can also be created at the translational level using aptamers, riboswitches, and ribozymes; this was recently reviewed by Ge and Marchisio (24).

In the present review, we describe how synthetic transcription-level genetic switches have been created in yeast. Synthetic genetic switches in yeast are categorized according 
to two types of regulation: a transcription activation/deactivation mode with synthetic transcription activators (sTAs) and a transcription repression/derepression mode with bare transcription repressors (Fig. 1). We also describe strategies to improve the switching performance of each genetic switch with different modes of action. Finally, we present the evolutionary techniques used to improve or create the functional genetic switches in yeast, especially in Saccharomyces cerevisiae.

(A) Activation/deactivation mode (Inducer-OFF)

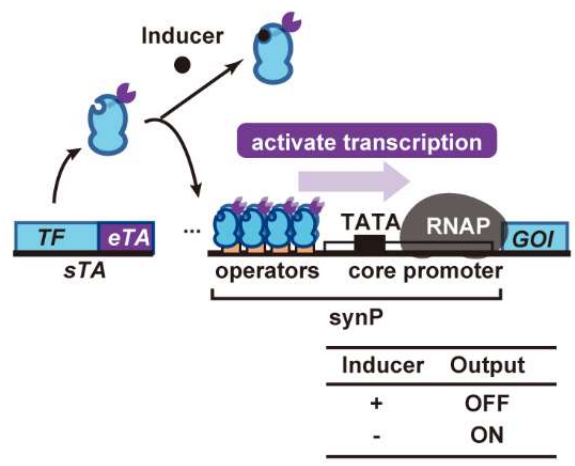

(B) Activation/deactivation mode (Inducer-ON)

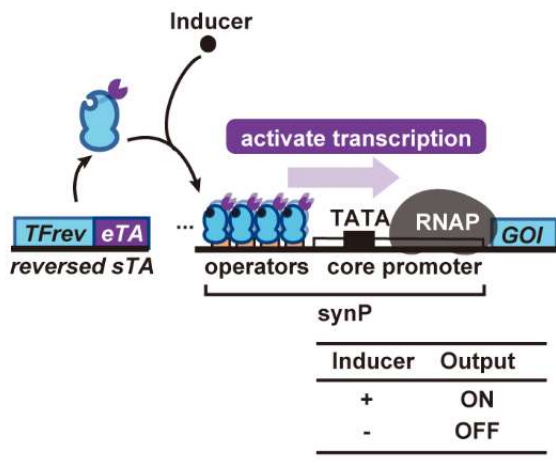

(C) Transcription activation without a eukaryotic activation motif (Inducer-ON)

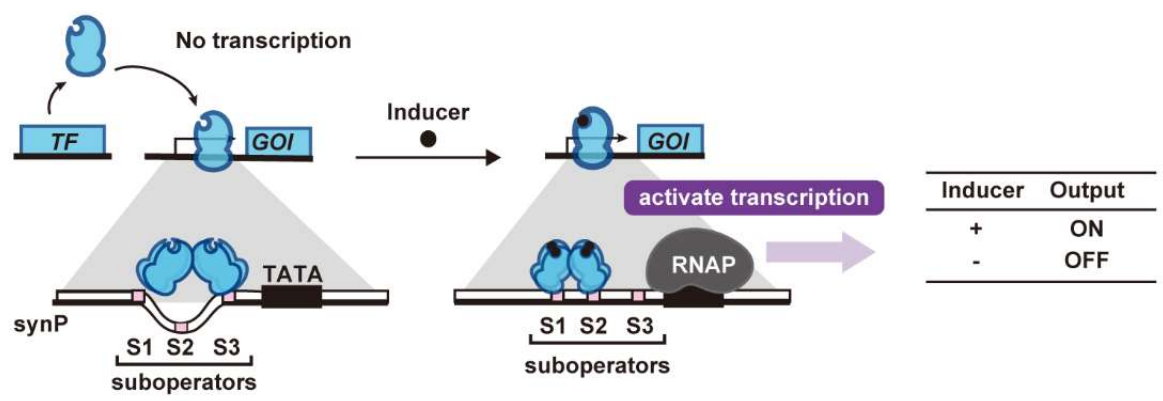

(D) Repression/derepression mode (Inducer-ON)

(E) Repression/derepression mode (Inducer-OFF)
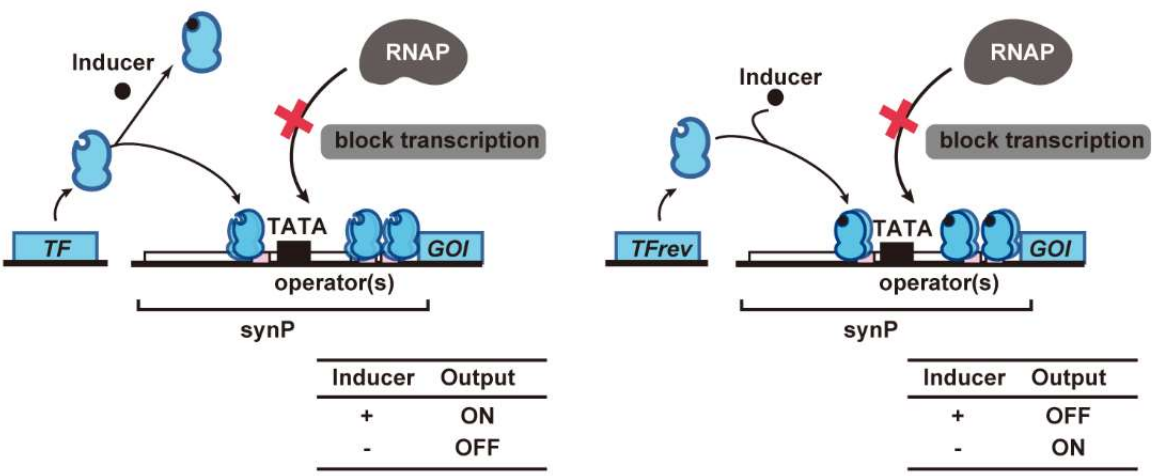

Figure 1. Construction of yeast genetic switches using synthetic transcriptional activators or bare transcription factors. Inducer-ON and inducer-OFF types of genetic switches can be constructed using sTA (A and B) and intact bacterial repressors (C, D and E), respectively. C. Transcription activation based on bacterial transcription activators. Ligand binding to transcription activators induces conformational changes to promoters and the recruitment of RNA polymerase. 
Abbreviations: sTA, synthetic transcription activator; TF, transcription factor; TFrev, reverse transcription factor; eTA, eukaryotic transcription activator; RNAP, RNA polymerase; TATA, TATA Box; synP, synthetic promoter; GOI, gene of interest.

\section{Synthetic transcriptional switches with different modes of regulation in yeast}

\subsection{Transcription Activation/Deactivation Mode}

Although transcription from a yeast promoter requires the recruitment of multiple endogenous TFs to the promoter, binding of a single protein fused with eukaryotic transcription activators (eTAs) is sufficient to artificially stimulate the recruitment of yeast TFs. Therefore, synthetic transcriptional switches can be created in yeast by fusing eTAs with ligand-responsive DNA binding proteins such as bacterial TFs (bTFs; also known as allosterically regulated TFs). With the appropriate design parameters, ligand-dependent binding of bTFs to their operator DNA sequences fused upstream of the yeast core promoter [i.e., a yeast promoter lacking an upstream activation sequence (UAS)] can be translated to the output gene expression (20, 23, 25, 26, 27) (Fig. 1A and 1B).

\subsection{1. sTAs Based on Transcription Repressors}

The Tet-OFF system $(22,23)$ is a famous and proven technology that uses a synthetic transcription activator, i.e., a tetracycline-controlled transactivator ( $\mathrm{tTA}$ ), to activate target gene expression. For example, a system incorporating a bacterial TetR repressor facilitates the control of strongly inducible expression in response to Dox, a more stable and less expensive analog of tetracycline (28). The tetracycline-responsive system has been used extensively as a synthetic genetic switch in gene circuits $(29,30,31,32)$; in particular, it has been used to explore the function of genes that confer toxic phenotypes because the switches provide regulated expression of the candidate genes $(33,34)$. Recently, variants of the tet $O$ sequence that binds to TetR with different affinities were developed by Fields' group as a toolkit for generating varying levels of gene expression (35). Using this toolkit, gene expression for carotenoid biosynthesis was tested and production of lycopene at high levels was successfully demonstrated.

Bacteria harbor various TetR homologues (36); the corresponding operator sequences and ligand molecules have been identified for some of these repressor proteins. A series of distant orthogonal genetic switches is a prerequisite to the independent and arbitrary regulation of multiple genes or pathways via different ligands. Boeke's group recently engineered distant homologues of the Escherichia coli TetR repressor from several species; thus, they developed new genetic switches that tightly control gene expression in S. cerevisiae $(26,27)$. In addition, Ikushima et al. have developed a genetic OFF switch that is tightly controlled in S. cerevisiae by camphor, an inexpensive small molecule (26). The transcriptional repressor CamR, a TetR homologue from Pseudomonas putida, binds to its operator sequence (camO) in the absence of camphor; however, it dissociates from the operator in response to the binding of the camphor ligand, which leads to the induction of the cytochrome P-450cam hydroxylase operon $(\operatorname{camDCAB})(37,38)$. In this case, an sTA was created by fusing three tandem repeats of the eTA (VP16) and a nuclear localization sequence (NLS) to the CamR transcriptional repressor. A corresponding synthetic promoter was also created by embedding six repeats of cam $O$ operator sequences between the $A D H 1$ terminator (to avoid leaky crosstalk from upstream transcription) and the CYC1 minimal promoter (which lacks the UAS). The resulting system (the Camphor-OFF switch) facilitates camphor-dependent regulation of downstream gene expression via an "inducerOFF"-type sTA (Fig. 1A). In this system, the sTA promotes transcription in the absence of camphor because ligand-free $\mathrm{CamR}$ binds to the camO operator elements. In contrast, the transcription remains OFF in the presence of camphor because camphor-bound CamR dissociates from the camO elements. In subsequent research conducted by the same group (27), a series of "inducer-OFF"-type genetic switches that function in S. cerevisiae were created using different TetR homologue transcription repressors and their cognate opera- 
tor DNAs and ligands: the DAPG-OFF switch [PhlF/phlO from Pseudomonas and 2,4-diacetylphloroglucinol (DAPG) (27)] and the Cumate-OFF switch (CymR/cymO from P. putida and $p$-cumate). Recently, the same design was applied to the construction of a malonylCoA sensor based on the transcription repressor FapR from Bacillus subtilis both in S. cerevisiae (39) and the nonconventional yeast Komagataella phaffii (40). In addition, the design was used to construct a xylose sensor based on the transcription repressor XylR from Staphylococcus xylosus (39). Umeyama et al. reported a S-adenosylmethionine (SAM) biosensor in yeast (41). Upon binding SAM, the repressor protein MetJ from E. coli binds to its operator DNA, metO. Thus, a MetJ-based biosensor for SAM was created by fusing MetJ with eTA (B42), and by fusing met $O$ with the $C Y C 1$ promoter upstream of the TATA box to create a synthetic promoter, namely metO-pCYC1.

Several eTAs, including VP16, B42, and Gal4, have been chosen for sTAs. Although many synthetic transcription activators have been constructed in yeast, as described above, eukaryotic activation motifs were not systematically compared until recently. In 2020, Qiu et al. compared the different transcription activation motifs [i.e., Gal4, Med2, VP16, VP64-p65-Rta (VPR), and Med2-Gal4] fused to the FapR repressor in terms of their activation capacities; they found that Med2 outperformed the other motifs in S. cerevisiae (39).

\subsection{2. sTAs Based on Ligand-induced DNA Binders}

Mutant sTAs can be used to create genetic switches with reversed switching behavior (Fig. 1B). The first report of a reversed sTA was a reversed tTA (rtTA) based on reversed Tet $R$ that binds to tetO in the presence of Dox. The discovery and evolutionary engineering of rtTA have been reviewed previously (28). The rtTA was first found in the directed evolution of E. coli (20) and then found in yeast (42). Subsequently, improved Dox-sensitivity was achieved using directed evolution in yeast (42) and then using viral evolution (43). In addition, Roney et al. serendipitously discovered a mutation in rtTA that significantly reduces leaky activation of rtTA (44). Recently, using a novel directed evolution platform described later (45) (section 3.4), we found a reverse PhlF mutant (rPhlF: PhlF with K86T, Q117R, and E143K) that binds to phlO in the presence of DAPG. We also found that the Q117R mutation in PhlF causes the reversed phenotype. A switch utilizing a synthetic transcription activator that was developed using this mutant PhIF showed $>100$-fold induction upon DAPG addition, which is comparable to the effect of a well-optimized TetON system.

In addition to mutant repressors, bacterial transcriptional activators are known to be ligand-triggered DNA binders. Moser et al. first described a synthetic biosensor that could sense methylating compounds, such as methylnitronitrosoguanidine and methyl methanesulfonate, in yeast (46). In this system, the N-terminal region of the Ada from E. coli was fused with the Gal4 transcription activation domain. Upon addition of methylating compounds, Ada protein is methylated to bind to the cognate operator sequence, which facilitates the transcriptional activation of the synthetic promoter. Later, Castano-Cerezo et al. engineered a 4-hydroxybenzoate responsive transcription activator, $\mathrm{HbaR}$, from Rhodopseudomonas palustris into an sTA by fusing the protein with a transcription activator, B12, and DNA binding protein, LexA (47). Wei et al. reported the fusion of a xylose responsive transcription activator, $\mathrm{XylR}$, from $E$. coli with a eukaryotic transcription activation motif, VPR, or heat shock factor 1 to create a xylose sensor both in S. cerevisiae and the oleic yeast Yarrowia lipolytica (48). In a recent example, we described the first yeast sTA based on a bacterial quorum-sensor protein, namely LuxR (45). Specifically, the TetR-family transcription activator LuxR was fused to three copies of VP16 (VP48) to activate a synthetic promoter in yeast composed of the GAL1 core promoter fused with 1-15 copies of the LuxR binding sequence (luxO). This system exhibited $>100$-fold induction upon addition of a quorum signal, i.e., 3-oxo-hexanoyl homoserine lactone (HSL). Notably, using directed evolution of LuxR to improve its sensitivity toward HSL in yeast enabled the identification of a novel sensitizing mutation that abolishes the native function of LuxR as 
a transcriptional activator in bacteria. This mutation would not be found during evolutionary engineering in a prokaryotic system where the transcriptional activation of LuxR is essential for the function of a genetic switch.

\subsection{3 sTAs Based on Ligand-responsive Nuclear Localizers}

sTAs can also be created with eukaryotic receptor proteins $(49,50,51,52,53)$. In one example, Chockalingam et al. demonstrated an sTA based on human estrogen receptor fused with both a DNA binding protein and transcription activation motif (49). In the absence of its native ligand, $\beta$-estrogen (including $\beta$-estradiol), the host Hsp90 chaperon complex binds to the sTA and prevents it from being transported to the nucleus (i.e., it remains in the cytosol). Upon ligand binding, the sTA is released from Hsp90 and transported into the nucleus to bind to the target DNA sequence; this results in the activation of target gene transcription. These authors mutated the ligand binding domain of the receptor to completely alter ligand specificity. Four rounds of saturation mutagenesis to the ligand binding domain and one round of whole-gene mutagenesis followed by genetic selection and screening enabled the authors to identify the mutant that was specifically responsive to the non-native ligand 4,4'-dihydroxybenzil (DHB). More recently, Mormino et al. developed a sTA based on the fusion of an acetic acid responsive transcription factor from S. cerevisiae, Haa1, and a DNA binding protein, BM3R1, from Bacillus megaterium (54). In this system, Haa1p is relocated to the nucleus following the binding of acetic acid, which causes binding to the BM3R1 binding sequence fused upstream of the yeast promoter. With this acetate-responsive genetic switch, it is possible to monitor the acetic acid concentration in yeast within a linear range from 10 to $60 \mathrm{mM}$.

\subsubsection{Transcription Activation Without a Eukaryotic Activation Motif}

Skjoedt et al. built biosensors based on bacterial LysR-type transcriptional regulators (Fig. 1C) (55). For systematic prototyping, they used BenM from Acinetobacter sp. ADP1, which responds to cis,cis-muconic acid (CCM), and its cognate operator DNA, benO. In the native system, BenM homotetramer binds to its cognate suboperators [sites 1 and 3 (S1 and S3)] even in the absence of CCM. When CCM binds to BenM, CCM-bound BenM homotetramer binds to different suboperators [sites 1 and 2 (S1 and S2)], which alters the DNA conformation and thereby enhances RNA polymerase (RNAP) binding to the bacterial promoter. Three synthetic promoters were created by fusing the yeast CYC1 promoter with a single ben $\mathrm{O}$ at different positions; one of the fusions with ben $\mathrm{O}$ at position -106 (upstream of the TATA box) resulted in a 20-fold increase in promoter activation upon constitutive expression of BenM from the TEF1 promoter. Surprisingly, this activation did not require the presence of a eukaryotic activation motif. With an evolutionarily optimized BenM mutant (H110R, F211V, and Y286N), cells exhibit a 10-fold increase in induction following the addition of 1.4-mM CCM. This synthetic promoter configuration has been used to construct three different biosensors by using the operators of FdeR ( $f d e O)$, $\operatorname{ArgP}(\operatorname{argO}), \mathrm{MdcR}(m d c O)$, and $\mathrm{PcaQ}(p c a O)$ instead of benO, which respond to naringenin, L-arginine, and malonic acid, protocatechuic acid, respectively $(55,56)$. In subsequent research by the same group, using fluorescent-activated cell sorting (FACS)aided directed evolution of BenM enabled the identification of a BenM mutants with reversed switching phenotype (CCM-induced deactivation; CCM-OFF), improved induction-fold and operational range, and altered ligand specificity (16).

\subsection{Transcription Repression/Derepression Mode}

When an intact bacterial transcription repressor binding sequence is fused to a position upstream of the TATA-box in a yeast promoter, transcription is blocked by inhibition of RNAP complex binding. The earliest example of this is a Dox-inducible system based on TetR and tet $O$, namely Tet-ON, in which intact TetR binds to tet $O$ and hinders the binding of RNAP to the promoter (transcription repression). Following the binding of Dox 
to TetR, the latter dissociates from the operator, resulting in the derepression of the synthetic promoter (Fig. 1D). With the aim of generating a diversity of promoters to enable fine-tuning of expression, the Ellis laboratory created a similar inverter device by placing the TetR regulatory machinery under the control of the highly expressed constitutive PFY1 promoter, which was identified using bioinformatics (57). Any transcription repressor and its binding target sequence from bacteria can be assembled into this type of genetic switch. Ikushima and Boeke demonstrated repressor-based sensing using the native PhlF repressor without the VP16 transcriptional activation domain (the DAPG-ON switch) (27) (Fig. 1E; the "inducer-ON"-type transcriptional repressor). One or two phlO elements were embedded downstream of the constitutive $A D H 1$ promoter and then the NLS-attached PhlF was constitutively expressed in yeast. In the absence of DAPG, ligandfree PhlF binds to the phlO elements (without DAPG), which represses reporter transcription; in the presence of DAPG, DAPG-bound PhlF dissociates from the phlO elements, which permits the initiation of reporter transcription. In principle, it should be possible to create an "inducer-OFF"-type transcriptional repressor using, for example, a mutant transcriptional repressor (rtTA and $\mathrm{rPhlF}$ ) exhibiting opposing binding behavior (as described in section 2.1.2).

D'Ambrosio et al. built a vanillin-responsive yeast genetic switch based on a vanillinresponsive transcriptional regulator, vanR from Caulobacter crescentus using systematic promoter engineering and FACS-assisted directed evolution; this switch enabled up to 3fold induction in the presence of 4-mM vanillin (15). In another study from the same group, Ambri et al. screened over 300 promoter designs to identify the optimal positions in synthetic promoters for three different bTF-binding sites (benO, vanO, and pcaO for BenM, VanR, and PcaQ, respectively) at single-nucleotide resolution in S. cerevisiae; the optimal insertion position was highly dependent on the type of bTF (56). Nevertheless, it has been possible to create such synthetic systems using the various inducer responsive transcription factor, XylR $(58,59,60)$, FdeR (61), FadR (62), and FapR (63), which respond to xylose, naringenin, fatty acids, and malonyl-coA, respectively. For XylR, the choice of bTF-operator combinations substantially affected the switching performance; following xylose addition, the maximum fold induction was obtained with a XylR/operator pair from B. subtilis (59). Furthermore, the same design has already be applied to different yeasts, e.g., fission yeast (64) and the methylotrophic yeast $K$. phaffii (65). In the latter instance, Cao et al. inserted the LacI binding sequence $(\mathrm{lacO})$ downstream of the GAPDH promoter, which resulted in a 6-fold induction of gene expression when isopropyl- $\beta$-D-thiogalactopyranoside (IPTG) was added (65). Recently, Gita et al. reported LacI/lacO-based regulation in combination with an artificial transcription activator based on a plant-derived TF; this system resulted in up to a 63-fold induction upon IPTG addition in K. phaffii (66).

To reduce the leakiness of regulated promoters, chromatin remodeling modules, such as Ssn6, can be fused to TFs. The effectiveness of this method was verified by constructing genetic switches with XylR-Ssn6 (60) and TetR-Ssn6 (22) fusion. Recently, Chen et al. performed rule-based optimization of this type of genetic switch (10). First, they investigated the minimal synthetic promoter sequence that had strong activity independent of nutrient conditions; the promoter with a 20-bp poly(T) sequence $4 \mathrm{bp}$ upstream of the TATA box met these criteria. They also found that blocking transcription readthrough by placing appropriate yeast terminators and ribozymes upstream of the regulated promoters could minimize the leakiness of the repressible genetic switches. Besides, the tightest repression by bTF was found to be achieved by separating two operators more than $20 \mathrm{bp}$. Using these design strategies, they developed three strongly repressible switches with $>100$-fold induction and low basal output. Furthermore, they applied the resultant genetic switches with a wide-dynamic range to automatically assemble yeast genetic circuits.

\section{Directed Evolution of Genetic Switches in Yeast}

As described above, any ligand-responsive DNA binding protein and its binding sequence can be assembled with yeast promoters to build synthetic gene regulation systems 
in yeast; however, the prototype switches often perform poorly. Researchers must identify the appropriate expression level of the sTAs or repressors by changing promoters and terminators (44). The optimal operator number and positions should be screened in a single base resolution $(15,25)$. The choice of yeast promoter sequence used to construct synthetic promoters via operator fusion inside/outside the promoters can substantially affect their performance $(56,59,67)$. The tuning process of eukaryotic genetic switches is far more difficult than the process required for prokaryotic cells because the behaviors of eukaryotic promoters are easily affected in an unpredictable manner by changes to surrounding sequences $(68,69)$. For example, the inducer sensitivity of a genetic switch may be altered by several orders of magnitude when it is transferred into different contexts (70); thus, to achieve desired performance levels, eukaryotic switches require extensive effort over many years to fine-tune expression and/or protein-engineer sTAs as well as to rearrange the sequence of the synthetic promoter, which was the case for the commonly used Tet-ON/Tet-OFF systems $(23,25,28)$.

Directed evolution is one of the tractable strategies used to develop useful transcriptional controllers in yeast. Genetic switches with desired performances are designed to be selected from the genetic switch library with randomized components. This strategy has been widely used in prokaryotic systems, especially in $E$. coli, resulting in a large genetic switch toolbox. In contrast, only a few examples of this evolutionary engineering strategy have been reported in yeast, despite it being routinely employed in E. coli $(1,71,72,73,74$, $75,76,77)$. In the following subsections, we summarize the recent successes in the evolutionary engineering of genetic switches in yeast as well as the methodologies used in each example.

\subsection{Fluorescence-based Screening}

Most genetic switches have been evaluated by placing genes for fluorescent protein as an output. Fluorescence measurements are then taken using multiwell-based plate readers or flow cytometry. Ellis et al. developed a tetracycline-responsive genetic switch in yeast by fusing the GAL1 promoter with two distinct tetO sequences where the TetR protein binds to repress transcription from the GAL1 promoter (78). They found switch variants with the optimal characteristics for construction of different genetic circuits (a feed-forward loop and timer) by performing green fluorescent protein (GFP)-based screening of synthetic promoter variants for which the sequence around the two tetOs were randomly mutated. Urlinger et al. performed GFP-based screening of approximately 1,000 clones on plates with and without Dox with the aim of identifying tTA mutants with a reversed phenotype (42). Furthermore, they performed a second round of mutagenesis and screening to identify rtTA mutants with improved sensitivity. As discussed in a previous review article (79), GFP-based screening is particularly important when the cells show clonal populations. FACS-based screening, rather than agar-plate or plate readerbased screening, can be essential.

\subsection{ON/OFF Selections Using FACS}

FACS enables the enrichment of cells with desired output levels at given conditions of from $10^{3}$ to $10^{5}$ variants per second $(80,81,82,83)$. This allows for high-throughput selection of vast libraries of genetic switches. With a FACS-based selection strategy, directed evolution of the responsivity of bacterial activators that are responsive to muconate (BenM) $(16,55)$ and vanillin (VanR) $(15)$ were performed to obtain mutants with the specifications described above (sections 2.1.4 and 2.2). Because FACS-based selection can be performed with tunable selection thresholds by changing gating conditions, it is independent of the specifications of prototype sensors. However, selection efficiency is highly dependent on both selection and gating conditions. Thus, sorting experiments must be repeated until the correct selection/gating condition is achieved given the lack of a priori knowledge related to the necessary selection conditions for each system. 


\subsection{ON/OFF Selections Using Genetic Selections}

Positive and negative selection can be used to enrich genetic switches with defined outputs under ON and OFF conditions. Positive selection markers include auxotrophic markers [e.g., His3/3-aminotriazole (3-AT)] (84), antibiotics markers (e.g., Ble/Zeocin KanMX/G418) (85), and counter selectable markers (86) such as Ura3/5-fluoroorotic acid (5-FOA) (84) and herpes simplex virus thymidine kinase (hsvTK)/5-fluorodeoxyuridine (5FdU) (87). Directed evolution of genetic switches using genetic selection was first demonstrated by Chockalingam et al., who converted $17 \beta$-estradiol responsive human receptor into a synthetic nonsteroidal compound, namely DHB responsive receptor (50). The synthetic transcription activator based on the mutant hormone receptor could be grown on a medium lacking histidine in the presence of DHB. Later, Klauser et al. demonstrated the use of both ON/OFF selections to enrich functional translational switches (84). In their system, the product of HIS3, imidazole glycerol phosphate dehydratase, was gradually inhibited dependent on the concentration of its inhibitor, 3-AT. Titration of 3-AT concentrations enabled the enrichment of cells with higher outputs under the ON-state (ON selection). OFF selection was based on the URA3, coding orotidine 5-phosphate decarboxylase; in the presence of 5-FOA, the cells expressing more URA3 converted more 5FOA into the toxic compound 5-fluorouracil, which enabled the enrichment of cells without any leaky URA3 expression in the absence of the ligand.

\section{4. "Screening of Selection" Strategy}

Despite the successes described above, the evolutionary strategy has not been widely applied to genetic switches in yeast. As previously described (79), stringent selection can be performed when genetic switches to be selected outcompete other nonfunctional (always-ON and always-OFF) variants; however, it is more difficult to select for mutants with a distinct $\mathrm{ON}$-state output from the majority of variants with slightly weaker output or vice versa. In most cases, even low expression levels of ON and OFF selection markers are already sufficient to allow cell growth and to cause cell death, respectively, which results in low selection efficiency. Moreover, genetic selection fails to discriminate desired genetic switches from others when their output levels under selection are too far off the given selection threshold $(84,88)$. Thus, it is important to identify the appropriate selection conditions that selectively enrich rare mutants with slightly improved performance. To meet this challenge, researchers must reconstruct the selector systems in almost every directed evolution cycle. However, even though the selection pressure of the His3/3-AT system is tunable by altering 3-AT concentration, reconstruction of the selection system is required to further optimize the selection pressure in some cases $(84,88)$. FACS-based selection is more capable of fine-tuning selection pressure, but the lack of a priori knowledge related to appropriate gating conditions for each system is a major obstacle to improving the application of this strategy.

One possible solution to this problem would involve performing multiple selections for different selection pressures in parallel and choosing the most promising selection pools from which the improved mutants would be screened (i.e., "screening of selection conditions"). We recently described an evolutionary platform for yeast genetic switches that could meet this demand we used a trifunctional fusion protein, consisting of hsvTK, Zeocin-resistance protein (Ble), and GFP (45). This fusion protein, hsvTK-Ble-GFP facilitates seamless ON/OFF selections with different selection pressures and only liquid handling in parallel using multiwell plates. Among the liquid-based ON selections, the combination of Ble and Zeocin is intrinsically suitable for genetic switches because the Ble dimer inactivates Zeocin by sequestering it from DNA at a 1:1 molar ratio; thus, the survival rate in the presence of Zeocin resistance of a cell should directly reflect the expression level of Ble. For OFF selection, we found that using hsvTK and the synthetic nucleoside 5FdU enables a progressive decrease in cell viability in proportion to the genetic switch output. The ON/OFF-selected cell populations can be characterized seamlessly us- 
ing flow cytometry, by which promising populations can be identified, from which improved genetic switch mutants can be screened. As a demonstration of this platform, we were able to isolate Dox-ON switches with improved fold induction ability by performing 14 selection conditions in parallel from which two promising conditions were subjected to further screening. Furthermore, we used the platform to develop a series of novel genetic switches with different specifications, namely DAPG-ON and HSL-ON switches (as described in section 2.1.1).

\section{Conclusions}

As described in this review, a number of bacterial TFs can easily be assembled into yeast genetic switches with or without the use of an evolutionary strategy. This method is now becoming the gold-standard for the engineering of genetic switches in yeast; however, rational engineering for the functional tuning of each component, which is crucial for maximizing genetic switch performance, is not feasible to date. Because the evolutionary platform for genetic switches in yeast has recently been demonstrated $(15,45,55)$, the number of such synthetic switches has rapidly increased, which highlights the potential of prokaryotic TFs as an untapped resource for yeast genetic switches. Besides, Umeno et al. argued that TFs are intrinsically evolvable; therefore, mutants that are responsive to non-native compounds can be reached within a few directed evolution cycles (89). Indeed, dozens of new or evolved genetic switches (biosensors) based on bacterial TFs have been reported within the last 2 years $(1,90,91,92,93,94,95,96,97,98)$. Library creation guided by machine-learning technology combined with deep mutagenesis and extensive sequencing technology will further accelerate the discovery of novel TF mutants (98). TFs created in this manner will be applied to yeast and then further evolved in yeast, possibly with the aid of in vivo autonomous mutagenesis techniques $(99,100,101)$ as well as automated continuous evolution technologies (102).

The genetic switches of yeast that can sense intracellular metabolites, i.e., metabolite sensors, are beginning to be used in high-throughput screening of higher metabolite-producing cells $(103,104)$ and in the metabolite-responsive dynamic control of enzyme expression in yeast $(105)$ (which was also recently reviewed $(106,107))$. These applications require that genetic switches can be optimized (evolved) depending on their purpose. For example, continuous evolution of cis,cis-muconic acid production in yeast uses the evolved sensory protein BenM (105). Besides, because yeast genetic switches and their assembly (genetic circuits) are based on bacterial TFs optimized in yeast, they are readily transferable into mammalian systems $(53,108)$. Expansion of the well-optimized (evolved) yeast genetic switches reviewed herein will result in the accelerated development of more complex mammalian systems. Taken together, directed evolution platforms for genetic switches in yeast can be one of the key technologies that push forward eukaryotic synthetic biology.

Author Contributions: Writing: M.T. and J. I., and A. K. All authors have read and agreed to the published version of the manuscript.

Funding: This research was partially funded by JSPS KAKENHI (grant number: 18K14374), the JST CREST program (grant number: JPMJCR21N2), and the JST-Mirai Program (grant number: JPMJMI17EJ) from Japan.

Institutional Review Board Statement: Not applicable.

Informed Consent Statement: Not applicable.

Conflicts of Interest: The authors declare no conflict of interest. 
1. Meyer, A. J., Segall-Shapiro, T. H., Glassey, E., Zhang, J., and Voigt, C. A.: Escherichia coli "Marionette" strains with 12 highly optimized small-molecule sensors, Nat Chem Biol, 15, 196-204 (2019).

2. Voigt, C. A.: Genetic parts to program bacteria, Curr Opin Biotechnol, 17, 548-557 (2006).

3. Douglas, H. C. and Hawthorne, D. C.: Enzymatic Expression and Genetic Linkage of Genes Controlling Galactose Utilization in Saccharomyces, Genetics, 49, 837-844 (1964).

4. Bassel, J. and Mortimer, R.: Genetic order of the galactose structural genes in Saccharomyces cerevisiae, J Bacteriol, 108, 179-183 (1971).

5. Ahn, J., Park, K. M., Lee, H., Son, Y. J., and Choi, E. S.: GAL promoter-driven heterologous gene expression in Saccharomyces cerevisiae Delta strain at anaerobic alcoholic fermentation, FEMS Yeast Res, 13, 140-142 (2013).

6. Vogl, T. and Glieder, A.: Regulation of Pichia pastoris promoters and its consequences for protein production, N Biotechnol, 30, 385-404 (2013).

7. Gander, M. W., Vrana, J. D., Voje, W. E., Carothers, J. M., and Klavins, E.: Digital logic circuits in yeast with CRISPR-dCas9 NOR gates, Nat Commun, 8, 15459 (2017).

8. Ryo, S., Ishii, J., Matsuno, T., Nakamura, Y., Matsubara, D., Tominaga, M., and Kondo, A.: Positive Feedback Genetic Circuit Incorporating a Constitutively Active Mutant Gal3 into Yeast GAL Induction System, ACS Synth Biol, 6, $928-935$ (2017).

9. Rantasalo, A., Kuivanen, J., Penttila, M., Jantti, J., and Mojzita, D.: Synthetic Toolkit for Complex Genetic Circuit Engineering in Saccharomyces cerevisiae, ACS Synth Biol, 7, 1573-1587 (2018).

10. Chen, Y., Zhang, S., Young, E. M., Jones, T. S., Densmore, D., and Voigt, C. A.: Genetic circuit design automation for yeast, Nat Microbiol, 5, 1349-1360 (2020).

11. Du, P., Zhao, H., Zhang, H., Wang, R., Huang, J., Tian, Y., Luo, X., Luo, X., Wang, M., Xiang, Y., and other 4 authors: De novo design of an intercellular signaling toolbox for multi-channel cell-cell communication and biological computation, Nat Commun, 11,4226 (2020).

12. Adeniran, A., Sherer, M., and Tyo, K. E. J.: Yeast-based biosensors: design and applications, Fems Yeast Research, 15 (2015).

13. D'Ambrosio, V. and Jensen, M. K.: Lighting up yeast cell factories by transcription factor-based biosensors, FEMS Yeast Res, 17 (2017).

14. Monteiro, F., Hubmann, G., Takhaveev, V., Vedelaar, S. R., Norder, J., Hekelaar, J., Saldida, J., Litsios, A., Wijma, H. J., Schmidt, A., and Heinemann, M.: Measuring glycolytic flux in single yeast cells with an orthogonal synthetic biosensor, Mol Syst Biol, 15, e9071 (2019).

15. D'Ambrosio, V., Pramanik, S., Goroncy, K., Jakočiūnas, T., Schönauer, D., Davari, M. D., Schwaneberg, U., Keasling, J. D., and Jensen, M. K.: Directed evolution of VanR biosensor specificity in yeast, Biotechnology notes, 2020 v.1, pp. 9-15 (2020).

16. Snoek, T., Chaberski, E. K., Ambri, F., Kol, S., Bjorn, S. P., Pang, B., Barajas, J. F., Welner, D. H., Jensen, M. K., and Keasling, J. D.: Evolution-guided engineering of small-molecule biosensors, Nucleic Acids Res, 48, e3 (2020).

17. Caliando, B. J. and Voigt, C. A.: Targeted DNA degradation using a CRISPR device stably carried in the host genome, Nat Commun, 6, 6989 (2015).

18. Brophy, J. A. N. and Voigt, C. A.: Principles of genetic circuit design, Nat Methods, 11, 508-520 (2014).

19. Shaw, W. M., Yamauchi, H., Mead, J., Gowers, G. F., Bell, D. J., Oling, D., Larsson, N., Wigglesworth, M., Ladds, G., and Ellis, T.: Engineering a Model Cell for Rational Tuning of GPCR Signaling, Cell, 177, 782-796 e727 (2019).

20. Gossen, M., Bonin, A. L., Freundlieb, S., and Bujard, H.: Inducible gene expression systems for higher eukaryotic cells, Curr Opin Biotechnol, 5, 516-520 (1994).

21. Helmschrott, C., Sasse, A., Samantaray, S., Krappmann, S., and Wagener, J.: Upgrading fungal gene expression on demand: improved systems for doxycycline-dependent silencing in Aspergillus fumigatus, Appl Environ Microbiol, 79, 1751-1754 (2013).

22. Belli, G., Gari, E., Piedrafita, L., Aldea, M., and Herrero, E.: An activator/repressor dual system allows tight tetracycline-regulated gene expression in budding yeast, Nucleic Acids Res, 26, 942-947 (1998). 
23. Gari, E., Piedrafita, L., Aldea, M., and Herrero, E.: A set of vectors with a tetracycline-regulatable promoter system for modulated gene expression in Saccharomyces cerevisiae, Yeast, 13, 837-848 (1997).

24. Ge, H. and Marchisio, M. A.: Aptamers, Riboswitches, and Ribozymes in S. cerevisiae Synthetic Biology, Life (Basel), 11 (2021).

25. Loew, R., Heinz, N., Hampf, M., Bujard, H., and Gossen, M.: Improved Tet-responsive promoters with minimized background expression, BMC Biotechnol, 10, 81 (2010).

26. Ikushima, S., Zhao, Y., and Boeke, J. D.: Development of a Tightly Controlled Off Switch for Saccharomyces cerevisiae Regulated by Camphor, a Low-Cost Natural Product, G3 (Bethesda), 5, 1983-1990 (2015).

27. Ikushima, S. and Boeke, J. D.: New Orthogonal Transcriptional Switches Derived from Tet Repressor Homologues for Saccharomyces cerevisiae Regulated by 2,4-Diacetylphloroglucinol and Other Ligands, ACS Synth Biol, 6, 497-506 (2017).

28. Das, A. T., Tenenbaum, L., and Berkhout, B.: Tet-On Systems For Doxycycline-inducible Gene Expression, Curr Gene Ther, 16, 156-167 (2016).

29. Becskei, A., Seraphin, B., and Serrano, L.: Positive feedback in eukaryotic gene networks: cell differentiation by graded to binary response conversion, EMBO J, 20, 2528-2535 (2001).

30. Nevozhay, D., Adams, R. M., Murphy, K. F., Josic, K., and Balazsi, G.: Negative autoregulation linearizes the dose-response and suppresses the heterogeneity of gene expression, Proc Natl Acad Sci U S A, 106, 5123-5128 (2009).

31. Zhuravel, D., Fraser, D., St-Pierre, S., Tepliakova, L., Pang, W. L., Hasty, J., and Kaern, M.: Phenotypic impact of regulatory noise in cellular stress-response pathways, Syst Synth Biol, 4, 105-116 (2010).

32. Gonzalez, C., Ray, J. C., Manhart, M., Adams, R. M., Nevozhay, D., Morozov, A. V., and Balazsi, G.: Stress-response balance drives the evolution of a network module and its host genome, Mol Syst Biol, 11, 827 (2015).

33. Boyer, J., Badis, G., Fairhead, C., Talla, E., Hantraye, F., Fabre, E., Fischer, G., Hennequin, C., Koszul, R., Lafontaine, I., and other 5 authors: Large-scale exploration of growth inhibition caused by overexpression of genomic fragments in Saccharomyces cerevisiae, Genome Biol, 5, R72 (2004).

34. Tabuchi, M., Kawai, Y., Nishie-Fujita, M., Akada, R., Izumi, T., Yanatori, I., Miyashita, N., Ouchi, K., and Kishi, F.: Development of a novel functional high-throughput screening system for pathogen effectors in the yeast Saccharomyces cerevisiae, Biosci Biotechnol Biochem, 73, 2261-2267 (2009).

35. Cuperus, J. T., Lo, R. S., Shumaker, L., Proctor, J., and Fields, S.: A tetO Toolkit To Alter Expression of Genes in Saccharomyces cerevisiae, ACS Synth Biol, 4, 842-852 (2015).

36. Cuthbertson, L. and Nodwell, J. R.: The TetR Family of Regulators, Microbiol Mol Biol R, 77, 440-475 (2013).

37. Aramaki, H., Sagara, Y., Hosoi, M., and Horiuchi, T.: Evidence for autoregulation of camR, which encodes a repressor for the cytochrome P-450cam hydroxylase operon on the Pseudomonas putida CAM plasmid, J Bacteriol, 175, 7828-7833 (1993).

38. Fujita, M., Aramaki, H., Horiuchi, T., and Amemura, A.: Transcription of the cam operon and camR genes in Pseudomonas putida PpG1, J Bacteriol, 175, 6953-6958 (1993).

39. Qiu, C., Chen, X., Rexida, R., Shen, Y., Qi, Q., Bao, X., and Hou, J.: Engineering transcription factor-based biosensors for repressive regulation through transcriptional deactivation design in Saccharomyces cerevisiae, Microbial Cell Factories, 19, 146 (2020).

40. Wen, J., Tian, L., Xu, M., Zhou, X., Zhang, Y., and Cai, M.: A Synthetic Malonyl-CoA Metabolic Oscillator in Komagataella phaffii, ACS Synth Biol, 9, 1059-1068 (2020).

41. Umeyama, T., Okada, S., and Ito, T.: Synthetic gene circuit-mediated monitoring of endogenous metabolites: identification of GAL11 as a novel multicopy enhancer of s-adenosylmethionine level in yeast, ACS Synth Biol, 2, $425-430$ (2013).

42. Urlinger, S., Baron, U., Thellmann, M., Hasan, M. T., Bujard, H., and Hillen, W.: Exploring the sequence space for tetracyclinedependent transcriptional activators: novel mutations yield expanded range and sensitivity, Proc Natl Acad Sci U S A, 97, 79637968 (2000).

43. Das, A. T., Zhou, X., Vink, M., Klaver, B., Verhoef, K., Marzio, G., and Berkhout, B.: Viral evolution as a tool to improve the tetracycline-regulated gene expression system, J Biol Chem, 279, 18776-18782 (2004). 
44. Roney, I. J., Rudner, A. D., Couture, J. F., and Kaern, M.: Improvement of the reverse tetracycline transactivator by single amino acid substitutions that reduce leaky target gene expression to undetectable levels, Sci Rep, 6, 27697 (2016).

45. Tominaga, M., Nozaki, K., Umeno, D., Ishii, J., and Kondo, A.: Robust and flexible platform for directed evolution of yeast genetic switches, Nat Commun, 12, 1846 (2021).

46. Moser, F., Horwitz, A., Chen, J., Lim, W., and Voigt, C. A.: Genetic sensor for strong methylating compounds, ACS Synth Biol, 2, 614-624 (2013).

47. Castano-Cerezo, S., Fournie, M., Urban, P., Faulon, J. L., and Truan, G.: Development of a Biosensor for Detection of Benzoic Acid Derivatives in Saccharomyces cerevisiae, Front Bioeng Biotechnol, 7, 372 (2019).

48. Wei, W., Shang, Y., Zhang, P., Liu, Y., You, D., Yin, B., and Ye, B.: Engineering Prokaryotic Transcriptional Activator XylR as a Xylose-Inducible Biosensor for Transcription Activation in Yeast, ACS Synth Biol, 9, 1022-1029 (2020).

49. Sanseverino, J., Gupta, R. K., Layton, A. C., Patterson, S. S., Ripp, S. A., Saidak, L., Simpson, M. L., Schultz, T. W., and Sayler, G. S.: Use of Saccharomyces cerevisiae BLYES expressing bacterial bioluminescence for rapid, sensitive detection of estrogenic compounds, Appl Environ Microbiol, 71, 4455-4460 (2005).

50. Chockalingam, K., Chen, Z., Katzenellenbogen, J. A., and Zhao, H.: Directed evolution of specific receptor-ligand pairs for use in the creation of gene switches, Proc Natl Acad Sci U S A, 102, 5691-5696 (2005).

51. McIsaac, R. S., Gibney, P. A., Chandran, S. S., Benjamin, K. R., and Botstein, D.: Synthetic biology tools for programming gene expression without nutritional perturbations in Saccharomyces cerevisiae, Nucleic Acids Res, 42, e48 (2014).

52. Ottoz, D. S., Rudolf, F., and Stelling, J.: Inducible, tightly regulated and growth condition-independent transcription factor in Saccharomyces cerevisiae, Nucleic Acids Res, 42, e130 (2014).

53. Feng, J., Jester, B. W., Tinberg, C. E., Mandell, D. J., Antunes, M. S., Chari, R., Morey, K. J., Rios, X., Medford, J. I., Church, G. M., Fields, S., and Baker, D.: A general strategy to construct small molecule biosensors in eukaryotes, Elife, 4 (2015).

54. Mormino, M., Siewers, V., and Nygard, Y.: Development of an Haa1-based biosensor for acetic acid sensing in Saccharomyces cerevisiae, FEMS Yeast Res (2021).

55. Skjoedt, M. L., Snoek, T., Kildegaard, K. R., Arsovska, D., Eichenberger, M., Goedecke, T. J., Rajkumar, A. S., Zhang, J., Kristensen, M., Lehka, B. J., and other 4 authors: Engineering prokaryotic transcriptional activators as metabolite biosensors in yeast, Nat Chem Biol, 12, 951-958 (2016).

56. Ambri, F., D'Ambrosio, V., Di Blasi, R., Maury, J., Jacobsen, S. A. B., McCloskey, D., Jensen, M. K., and Keasling, J. D.: HighResolution Scanning of Optimal Biosensor Reporter Promoters in Yeast, ACS Synth Biol, 9, 218-226 (2020).

57. Blount, B. A., Weenink, T., Vasylechko, S., and Ellis, T.: Rational diversification of a promoter providing fine-tuned expression and orthogonal regulation for synthetic biology, PLoS One, 7, e33279 (2012).

58. Teo, W. S. and Chang, M. W.: Bacterial XylRs and synthetic promoters function as genetically encoded xylose biosensors in Saccharomyces cerevisiae, Biotechnol J, 10, 315-322 (2015).

59. Wang, M., Li, S., and Zhao, H.: Design and engineering of intracellular-metabolite-sensing/regulation gene circuits in Saccharomyces cerevisiae, Biotechnol Bioeng, 113, 206-215 (2016).

60. Hector, R. E. and Mertens, J. A.: A Synthetic Hybrid Promoter for Xylose-Regulated Control of Gene Expression in Saccharomyces Yeasts, Mol Biotechnol, 59, 24-33 (2017).

61. Wang, R. F., Cress, B. F., Yang, Z., Hordines, J. C., Zhao, S. J., Jung, G. Y., Wang, Z. T., and Koffas, M. A. G.: Design and Characterization of Biosensors for the Screening of Modular Assembled Naringenin Biosynthetic Library in Saccharomyces cerevisiae, Acs Synthetic Biology, 8, 2121-2130 (2019).

62. Teo, W. S., Hee, K. S., and Chang, M. W.: Bacterial FadR and synthetic promoters function as modular fatty acid sensor- regulators in Saccharomyces cerevisiae, Engineering in Life Sciences, 13, 456-463 (2013).

63. Li, S., Si, T., Wang, M., and Zhao, H.: Development of a Synthetic Malonyl-CoA Sensor in Saccharomyces cerevisiae for Intracellular Metabolite Monitoring and Genetic Screening, ACS Synth Biol, 4, 1308-1315 (2015). 
64. Kjaerulff, S. and Nielsen, O.: An IPTG-inducible derivative of the fission yeast nmt promoter, Yeast, 32, 469-478 (2015).

65. Cao, J., Perez-Pinera, P., Lowenhaupt, K., Wu, M. R., Purcell, O., de la Fuente-Nunez, C., and Lu, T. K.: Versatile and on-demand biologics co-production in yeast, Nat Commun, 9, 77 (2018).

66. Naseri, G., Prause, K., Hamdo, H. H., and Arenz, C.: Artificial Transcription Factors for Tuneable Gene Expression in Pichia pastoris, Front Bioeng Biotechnol, 9, 676900 (2021).

67. Ede, C., Chen, X., Lin, M. Y., and Chen, Y. Y.: Quantitative Analyses of Core Promoters Enable Precise Engineering of Regulated Gene Expression in Mammalian Cells, ACS Synth Biol, 5, 395-404 (2016).

68. Lee, T. J., Parikh, R. Y., Weitz, J. S., and Kim, H. D.: Suppression of Expression Between Adjacent Genes Within Heterologous Modules in Yeast, G3 Genes I Genomes I Genetics, 4, 109-116 (2014).

69. Redden, H. and Alper, H. S.: The development and characterization of synthetic minimal yeast promoters, Nat Commun, 6, 7810 (2015).

70. Neddermann, P., Gargioli, C., Muraglia, E., Sambucini, S., Bonelli, F., De Francesco, R., and Cortese, R.: A novel, inducible, eukaryotic gene expression system based on the quorum-sensing transcription factor TraR, EMBO Rep, 4, 159-165 (2003).

71. Muranaka, N., Sharma, V., Nomura, Y., and Yokobayashi, Y.: An efficient platform for genetic selection and screening of gene switches in Escherichia coli, Nucleic Acids Res, 37, e39 (2009).

72. Shong, J., Huang, Y. M., Bystroff, C., and Collins, C. H.: Directed evolution of the quorum-sensing regulator EsaR for increased signal sensitivity, ACS Chem Biol, 8, 789-795 (2013).

73. Ike, K., Arasawa, Y., Koizumi, S., Mihashi, S., Kawai-Noma, S., Saito, K., and Umeno, D.: Evolutionary Design of CholineInducible and -Repressible T7-Based Induction Systems, ACS Synth Biol, 4, 1352-1360 (2015).

74. Tashiro, Y., Kimura, Y., Furubayashi, M., Tanaka, A., Terakubo, K., Saito, K., Kawai-Noma, S., and Umeno, D.: Directed evolution of the autoinducer selectivity of Vibrio fischeri LuxR, J Gen Appl Microbiol, 62, 240-247 (2016).

75. Saeki, K., Tominaga, M., Kawai-Noma, S., Saito, K., and Umeno, D.: Rapid Diversification of BetI-Based Transcriptional Switches for the Control of Biosynthetic Pathways and Genetic Circuits, ACS Synth Biol, 5, 1201-1210 (2016).

76. De Paepe, B., Peters, G., Coussement, P., Maertens, J., and De Mey, M.: Tailor-made transcriptional biosensors for optimizing microbial cell factories, J Ind Microbiol Biotechnol, 44, 623-645 (2017).

77.Ellefson, J. W., Ledbetter, M. P., and Ellington, A. D.: Directed evolution of a synthetic phylogeny of programmable Trp repressors, Nat Chem Biol, 14, 361-367 (2018).

78. Ellis, T., Wang, X., and Collins, J. J.: Diversity-based, model-guided construction of synthetic gene networks with predicted functions, Nat Biotechnol, 27, 465-471 (2009).

79. Schaerli, Y. and Isalan, M.: Building synthetic gene circuits from combinatorial libraries: screening and selection strategies, Mol Biosyst, 9, 1559-1567 (2013).

80. Ibrahim, S. F. and van den Engh, G.: Flow cytometry and cell sorting, Adv Biochem Eng Biotechnol, 106, 19-39 (2007).

81. Becker, S., Schmoldt, H. U., Adams, T. M., Wilhelm, S., and Kolmar, H.: Ultra-high-throughput screening based on cell-surface display and fluorescence-activated cell sorting for the identification of novel biocatalysts, Curr Opin Biotechnol, 15, 323-329 (2004).

82. Yang, G. and Withers, S. G.: Ultrahigh-throughput FACS-based screening for directed enzyme evolution, Chembiochem, 10, 27042715 (2009).

83. Cossarizza, A., Chang, H. D., Radbruch, A., Acs, A., Adam, D., Adam-Klages, S., Agace, W. W., Aghaeepour, N., Akdis, M., Allez, M., and other 426 authors: Guidelines for the use of flow cytometry and cell sorting in immunological studies (second edition), Eur J Immunol, 49, 1457-1973 (2019).

84. Klauser, B., Atanasov, J., Siewert, L. K., and Hartig, J. S.: Ribozyme-based aminoglycoside switches of gene expression engineered by genetic selection in S. cerevisiae, ACS Synth Biol, 4, 516-525 (2015).

85. Johansson, B. and Hahn-Hagerdal, B.: Overproduction of pentose phosphate pathway enzymes using a new CRE-loxP expression vector for repeated genomic integration in Saccharomyces cerevisiae, Yeast, 19, 225-231 (2002). 
86. Gnugge, R. and Rudolf, F.: Saccharomyces cerevisiae Shuttle vectors, Yeast, 34, 205-221 (2017).

87. Alexander, W. G., Doering, D. T., and Hittinger, C. T.: High-efficiency genome editing and allele replacement in prototrophic and wild strains of Saccharomyces, Genetics, 198, 859-866 (2014).

88. Rugbjerg, P., Genee, H. J., Jensen, K., Sarup-Lytzen, K., and Sommer, M. O.: Molecular Buffers Permit Sensitivity Tuning and Inversion of Riboswitch Signals, ACS Synth Biol, 5, 632-638 (2016).

89. Umeno, D., Kimura, Y., and Kawai-Noma, S.: Transcription factors as evolvable biosensors, Analytical Sciences, advpub (2021).

90. L, F. M. M., Currin, A., and Dixon, N.: Directed evolution of the PcaV allosteric transcription factor to generate a biosensor for aromatic aldehydes, J Biol Eng, 13, 91 (2019).

91. Chouichit, P., Whangsuk, W., Sallabhan, R., Mongkolsuk, S., and Loprasert, S.: A highly sensitive biosensor with a single-copy evolved sensing cassette for chlorpyrifos pesticide detection, Microbiology (Reading), 166, 1019-1024 (2020).

92. Della Corte, D., van Beek, H. L., Syberg, F., Schallmey, M., Tobola, F., Cormann, K. U., Schlicker, C., Baumann, P. T., Krumbach, K., Sokolowsky, S., and other 5 authors: Engineering and application of a biosensor with focused ligand specificity, Nat Commun, 11,4851 (2020).

93. Jia, X., Ma, Y., Bu, R., Zhao, T., and Wu, K.: Directed evolution of a transcription factor PbrR to improve lead selectivity and reduce zinc interference through dual selection, AMB Express, 10, 67 (2020).

94. Kwon, K. K., Yeom, S. J., Choi, S. L., Rha, E., Lee, H., Kim, H., Lee, D. H., and Lee, S. G.: Acclimation of bacterial cell state for high-throughput enzyme engineering using a DmpR-dependent transcriptional activation system, Sci Rep, 10, 6091 (2020).

95. Sun, H., Zhao, H., and Ang, E. L.: A New Biosensor for Stilbenes and a Cannabinoid Enabled by Genome Mining of a Transcriptional Regulator, ACS Synth Biol, 9, 698-705 (2020).

96. Tang, R. Q., Wagner, J. M., Alper, H. S., Zhao, X. Q., and Bai, F. W.: Design, Evolution, and Characterization of a Xylose Biosensor in Escherichia coli Using the XylR/xylO System with an Expanded Operating Range, ACS Synth Biol, 9, 2714-2722 (2020).

97. Zhang, X., Cao, Y., Liu, Y., Liu, L., Li, J., Du, G., and Chen, J.: Development and optimization of N-acetylneuraminic acid biosensors in Bacillus subtilis, Biotechnol Appl Biochem, 67, 693-705 (2020).

98. Unger, E. K., Keller, J. P., Altermatt, M., Liang, R. Q., Matsui, A., Dong, C. Y., Hon, O. J., Yao, Z., Sun, J. Q., Banala, S., and other 25 authors: Directed Evolution of a Selective and Sensitive Serotonin Sensor via Machine Learning, Cell, 183, 1986-+ (2020).

99. Crook, N., Abatemarco, J., Sun, J., Wagner, J. M., Schmitz, A., and Alper, H. S.: In vivo continuous evolution of genes and pathways in yeast, Nat Commun, 7, 13051 (2016).

100. Ravikumar, A., Arzumanyan, G. A., Obadi, M. K. A., Javanpour, A. A., and Liu, C. C.: Scalable, Continuous Evolution of Genes at Mutation Rates above Genomic Error Thresholds, Cell, 175, 1946-1957 e1913 (2018).

101. Tou, C. J., Schaffer, D. V., and Dueber, J. E.: Targeted Diversification in the S. cerevisiae Genome with CRISPR-Guided DNA Polymerase I, ACS Synth Biol, 9, 1911-1916 (2020).

102. Zhong, Z., Wong, B. G., Ravikumar, A., Arzumanyan, G. A., Khalil, A. S., and Liu, C. C.: Automated Continuous Evolution of Proteins in Vivo, ACS Synth Biol, 9, 1270-1276 (2020).

103. Jensen, E. D., Ambri, F., Bendtsen, M. B., Javanpour, A. A., Liu, C. C., Jensen, M. K., and Keasling, J. D.: Integrating continuous hypermutation with high-throughput screening for optimization of cis,cis-muconic acid production in yeast, Microb Biotechnol (2021).

104. David, F., Nielsen, J., and Siewers, V.: Flux Control at the Malonyl-CoA Node through Hierarchical Dynamic Pathway Regulation in Saccharomyces cerevisiae, ACS Synth Biol, 5, 224-233 (2016).

105. Zhang, Y. and Shi, S.: Transcription Factor-Based Biosensor for Dynamic Control in Yeast for Natural Product Synthesis, Front Bioeng Biotechnol, 9, 635265 (2021).

106. Marsafari, M., Ma, J. B., Koffas, M., and Xu, P.: Genetically-encoded biosensors for analyzing and controlling cellular process in yeast, Curr Opin Biotech, 64, 175-182 (2020). 
107. Zhang, Y. M. and Shi, S. B.: Transcription Factor-Based Biosensor for Dynamic Control in Yeast for Natural Product Synthesis, Front Bioeng Biotech, 9 (2021).

108. Nevozhay, D., Zal, T., and Balazsi, G.: Transferring a synthetic gene circuit from yeast to mammalian cells, Nat Commun, 4 , 1451 (2013). 